\title{
FACTORES EDÁFICOS QUE INFLUYEN EN EL CRECIMIENTO DE Vochysia guatemalensis EN LA REGIÓN HUETAR NORTE DE COSTA RICA
}

\author{
Fernando Mora ${ }^{1 / *}$, Roger Muñoz ${ }^{* *}$, Víctor Meza*, William Fonseca*** \\ Palabras clave: Sebo; Mayo blanco; Barba chele; factores críticos; índice de sitio. \\ Keywords: Sebo; Mayo blanco; Barba chele; critical factors; index quality.
}

Recibido: 20/11/13

\section{RESUMEN}

Se evalúa el crecimiento de Vochysia guatemalensis en Ultisoles e Inceptisoles de la Región Huetar Norte de Costa Rica. Este documento presenta las condiciones de los sitios donde la especie está creciendo en la región bajo estudio. Por medio de variables físicas y químicas de los suelos, se explicó el comportamiento del índice de sitio (IS) y su relación con aquellas variables edáficas que muestran algún tipo de relación con este indicador de la calidad de los terrenos. Además, se determinaron 2 profundidades los factores del suelo que limitan o favorecen el crecimiento de esta especie, con el propósito de identificar tanto los sitios apropiados como los sitios inadecuados para la especie. A una profundidad de 0 a $20 \mathrm{~cm}$, los mejores sitios (IS mayor de 23), están relacionados con $\mathrm{pH}$ bajos, concentraciones bajas de calcio y concentraciones medias de magnesio, una suma de bases y una capacidad de intercambio catiónica efectiva medias, una acidez extraíble y una saturación de acidez media. De 20 a $40 \mathrm{~cm}$, se observa que los mejores sitios están asociados con una baja
Aceptado: 27/03/15

\begin{abstract}
Edaphic factors that influence the growth of Vochysia guatemalensis in the Northern Huetar Region of Costa Rica. The growth of Vochysia guatemalensis in Ultisols and Inceptisols of the Northern Huetar Region of Costa Rica were evaluated. This paper introduces the conditions of the sites, in the region under study where the species is growing. Making use of physical and chemical soil variables, it tries to explain the behavior of the site index (Is) and its relationship with those edaphic variables that show some kind of relation with this indicator of the quality of the land. In addition, working at 2 depths, the factors of the soil which limit or favor the growth of this species are determined, in order to identify the most appropriate, as well as the most inadequate, sites for the species. At to $20 \mathrm{~cm}$ depth, the best sites (IS greater than 23), are related to low $\mathrm{pH}$, low calcium concentrations and median concentrations of magnesium, medium sum of bases and medium effective cation exchange capacity, an extractable medium acidity and medium saturation acidity.
\end{abstract}

\footnotetext{
1 Autor para correspondencia. Correo electrónico: fernando.mora.chacon@una.cr

Instituto de Investigación y Servicios Forestales, Universidad Nacional, Heredia, Costa Rica.
} 
densidad aparente, un alto contenido de arcilla y de hierro y una buena cantidad de materia orgánica. Los suelos que mostraron mejor condición química, son los que presentan la peor calidad de sitio (IS menor de 20) para V. guatemalensis. Esto resalta los bajos requerimientos nutricionales que presenta la especie la cual puede considerarse como una especie adaptada a suelos relativamente ácidos, de baja fertilidad.

\section{INTRODUCCIÓN}

El éxito del cultivo de árboles se debe entre otras cosas, a la respuesta de la especie a la capacidad productiva de los sitios donde se va a plantar (Clutter et ál. 1983, Philip 1983, Davis y Johnson 1987). Esta capacidad productiva es definida por una amplia gama de factores ambientales fisiográficos, climáticos y edáficos que junto a la capacidad genética de la especie interactúan entre sí, para definir el desarrollo de la misma. Sin embargo, en algunos casos la presencia o ausencia de uno o más de estos factores ambientales puede llegar a actuar ya sea como factor limitante o bien, como un factor impulsador del crecimiento de una especie forestal, como es el caso de las especies del género Vochysia, que están bien adaptadas a los suelos ácidos y poco fértiles de la región tropical ya que acumulan aluminio en el follaje (Chenery y Sporne 1976, Foy et ál. 1978, citados por Pérez et ál. 1993), asî como Mn foliar (Badilla 2012).

En el caso de Costa Rica, una de las especies nativas más importantes usadas en la reforestación es $V$. guatemalensis, conocida como Chancho, Sebo, Mayo blanco o Barba chele (Flores 1993), que se encuentra distribuída naturalmente en toda la Región Huetar Norte donde se planta el $25 \%$ del total reforestado con esta
From 20 to $40 \mathrm{~cm}$, it is observed that the best sites are associated with low apparent density, a high content of clay and iron, and a good amount of organic matter. The soils that show the best chemical condition are those associated with the worst quality of site (IS lesser than 20) for $V$. guatemalensis. This highlights the low nutritional requirements of this species, $V$. guatemalensis, which can be considered as adapted to relatively acidic soils of low fertility.

especie en todo el país (Solís y Moya 2004). La especie es considerada muy versátil ya que se puede utilizar en prácticas silvícolas, como por ejemplo sistemas silvopastoriles (Moulaert et ál. 2002), sistemas agroforestales (Haggar et ál. 2003), plantaciones mixtas (Butterfield y Espinoza 1994, Montagnini y Sancho 1994) y plantaciones puras (Flores 1993, Di Stéfano y Fournier 1998, Solís y Moya 2004), lo cual aumenta significativamente su potencial en programas de reforestación (CAB International 2000). En atención a los factores edáficos, este documento presenta datos empíricos sobre el rol que ciertos factores del suelo tienen en el crecimiento de $V$. guatemalensis y se recomienda que estos sean tomados en cuenta para futuros programas de reforestación con la especie.

\section{Distribución natural y condiciones bioclimáticas}

Según CAB International (2000) V. guatemalensis se distribuye naturalmente en Norte América (México), en Centro América (Belize, Guatemala, Honduras, Nicaragua y Costa Rica) y en Sur América (Colombia). Adicionalmente, Alvarado y Raigosa (2012), amplían el rango de distribución hasta Perú. La especie se puede 
encontrar en elevaciones que van desde los 0 msnm hasta los 1000 msnm (Flores 1993).

Climatológicamente la región de origen presenta una precipitación media anual que oscila entre 600 y $3200 \mathrm{~mm}$, con una estación seca de hasta 6 meses (CAB International 2000). No obstante, Flores (1993) afirma que se puede encontrar en regiones donde la precipitación anual varía de 3000 a $5500 \mathrm{~mm}$. La región donde crece naturalmente la especie, presenta un rango de temperatura media anual que va de 12 a $35^{\circ} \mathrm{C}$, una temperatura media máxima del mes más caliente entre 23 y $38^{\circ} \mathrm{C}$, una temperatura media mínima del mes más frío entre 8 y $10^{\circ} \mathrm{C}$ y una temperatura mínima absoluta $>4^{\circ} \mathrm{C}$ (CAB International 2000).

Según (Flores 1993), la especie crece muy bien cerca de ríos y quebradas, en suelos formados sobre aluviones arenosos y en ocaciones en suelos desarrollados sobre materiales coluviales. También por su capacidad de adaptación le permite crecer en una variedad de condiciones como suelos ácidos (con pH de 5,0 a 6,0), con textura franco arcillosa a limosa y con altas concentraciones de óxidos e hidróxidos de hierro y aluminio (Pérez et ál. 1993).

\section{Relaciones suelo-sitio}

La calidad de sitio está definida por la combinación de un conjunto de factores ambientales (bióticos, climáticos, edáficos y fisiográficos) que influyen sobre el crecimiento de los árboles y de las masas forestales, así como sobre la capacidad de producción de los terrenos (Mora 1992, Prodan et ál. 1997). Debería ser posible determinar la calidad de un sitio en forma directa, al medir individualmente, todos los factores que afectan la productividad de los terrenos para luego integrarlos en algún tipo de índice global de clasificación (Mora 1986).

En investigaciones, se ha optado por utilizar 2 formas diferentes para evaluar esa capacidad de producción: a) a través de métodos directos o bien, b) mediante métodos indirectos. En el primer grupo se encuentra el indicador conocido como "índice de sitio", definido por
Prodan et ál. (1997) como "la altura dominante o predominante promedio alcanzada por un rodal o una parcela a una 'edad clave' determinada". En el segundo grupo encontramos los "estudios suelo-sitio", los cuales pretenden identificar la calidad de un lugar, bajo el concepto de que algunas características edáficas pueden estar correlacionadas con el índice de sitio.

El uso del índice de sitio como variable dependiente en la clasificación de los terrenos, en asocio con algunas variables del suelo (estudios suelo-sitio), está debidamente documentado en una serie de trabajos realizados desde los años 50 , cuando se incluyeron ciertos nutrimentos del suelo en algunas ecuaciones predictivas. De hecho, el contenido de $\mathrm{P}, \mathrm{Ca}$ y $\mathrm{K}$ en el horizonte $\mathrm{A}$, junto con el contenido de materia orgánica en el horizonte $\mathrm{B}$, fueron los factores del suelo más importantes que afectaron el crecimiento en altura de Eucalyptus alba en Brasil (Barros y Withmore 1980). En Colombia, Fassbender y Tschinkel (1974), encontraron que el índice de sitio de las plantaciones de Cupressus lusitanica, en suelos volcánicos, se vio afectado por el contenido de fosfatos de aluminio y por las cantidades de $\mathrm{K}$ y $\mathrm{Mg}$ intercambiables. El índice de sitio de Araucaria angustifolia demostró estar positivamente correlacionado con el porcentaje de saturación de bases del horizonte A (De Hoogh y Dietrich 1979). En Costa Rica, Alvarado y Fallas (2004) demostraron la sensibilidad de la Teca a la saturación de acidez del suelo, donde valores mayores a un 3,0\% reducen el incremento medio anual en altura significativamente. En resumen, la importancia relativa de los rasgos suelo-sitio para predecir la calidad de un lugar puede variar o ser diferente de acuerdo con la especie, el suelo, la topografía y el clima del área, como se señaló anteriormente. Por esta razón, los resultados son válidos solo para la especie en cuestión, bajo las condiciones estudiadas.

En el caso de $V$. guatemalensis, se desconoce hasta ahora, cuáles son los requerimientos óptimos del sitio y del suelo para esta especie (Solís y Moya 2004). Sin embargo, Di Stéfano y Fournier (1998), atribuyen el menor crecimiento 
de los árboles de $V$. guatemalensis en Tabarcia de Mora (al compararlo con el mayor crecimiento de los árboles en Sarapiquí y Guápiles), a las altas concentraciones de $\mathrm{P}$ en el suelo, en vista de que esta especie parece verse afectada por una presencia abundante de este elemento (Di Stéfano y Fournier 1994, Herrera 1996). Por su parte, Butterfield (1996) evaluó la tasa de mortalidad y la altura promedio para $V$. guatemalensis, correlacionándolas con ciertas variables químicas del suelo como, M.O., P, CICE, pH y saturación de Al. Se supone que las características edáficas, así como las diferencias entre los distintos órdenes del suelo, pueden influir positiva o negativamente en la distribución y crecimiento de esta especie. Arias et ál. (2011) muestran cómo la fertilidad de los suelos y la zona de vida afectan significativamente las variables de crecimiento de esta especie.

En este estudio se propone evaluar el crecimiento de $V$. guatemalensis en Ultisoles e Inceptisoles de la Región Huetar Norte. Se mencionan las condiciones de los sitios donde la especie se encuentra en crecimiento según la región estudiada; además con las variables físicas y químicas de los suelos, se explica el comportamiento del índice de sitio y su relación con aquellas variables edáficas que presentan algún tipo de relación con dicho indicador. Además, se propone determinar los factores del suelo que limitan o favorecen el crecimiento de esta especie, con la intención de identificar los sitios más apropiados para la misma.

\section{MATERIALES Y MÉTODOS}

\section{Localización y descripción del área de estudio}

La investigación se llevó a cabo entre entre setiembre y octubre del 2004 en la Región Huetar Norte de Costa Rica, que incluye los cantones de San Carlos, Los Chiles, Upala y Guatuso. Los 12 sitios de muestreo, incluidos en el presente estudio, se agruparon de la siguiente manera: 7 en el cantón de San Carlos (San Gerardo, El Jardín de Cutris (2), Betania (2), Buenos Aires y CoopeVega), 4 en el cantón de Los Chiles (Los Chiles (2), Escaleras y Montealegre) y 1 en el cantón de Upala (Chimurria); todos en la provincia de Alajuela.

La localización de los sitios de estudio, su ubicación geográfica, la información climática y sus respectivas zonas de vida se muestran en el Cuadro 1. El área de estudio se concentra en las siguientes hojas cartográficas, escala 1:50.000, Tres Amigos, Infiernito, Monterrey, San Jorge y Medio Queso, las cuales cubren un área de 250 $\mathrm{km}^{2}$, entre las latitudes $10^{\circ} 33^{\prime}$ y $10^{\circ} 58^{\prime}$ y entre las longitudes $84^{\circ} 18^{\prime}$ y $84^{\circ} 41^{\prime}$. 
Cuadro 1. Características generales de los 12 sitios muestreados en plantaciones de V. guatemalensis en Ultisoles e Inceptisoles, Zona Huetar Norte de Costa Rica.

\begin{tabular}{|c|c|c|c|c|c|c|}
\hline Finca & Símbolo & $\begin{array}{l}\text { Localidad } \\
\text { /Cantón }\end{array}$ & $\begin{array}{l}\text { Elevación } \\
(\mathrm{msnm}) \\
(*)\end{array}$ & $\begin{array}{l}\text { Ppt media } \\
\text { anual } \\
(\mathrm{mm})(*)\end{array}$ & $\begin{array}{l}\text { Zona de Vida } \\
(*)\end{array}$ & $\begin{array}{l}\text { Hoja cartográfica } \\
1: 50.000 \\
(*)\end{array}$ \\
\hline La Tite & LT & $\begin{array}{l}\text { San Gerardo, } \\
\text { San Carlos }\end{array}$ & 80 & 2201 & Bmh-P/TB & Tres Amigos 3347 I \\
\hline Oscar Rodríguez 2 & OR2 & $\begin{array}{l}\text { El Jardín, San } \\
\text { Carlos }\end{array}$ & 177 & 3200 & Bmh-T & Infiernito 3348 III \\
\hline Oscar Rodríguez 4 & OR4 & $\begin{array}{l}\text { El Jardín, San } \\
\text { Carlos }\end{array}$ & 119 & 3200 & Bmh-T & Infiernito 3348 III \\
\hline Miguel Valle & MV & $\begin{array}{l}\text { Buenos Aires, } \\
\text { San Carlos }\end{array}$ & - & 3200 & Bmh-T & Monterrey 3247 I \\
\hline Edwin Romero & ER & $\begin{array}{l}\text { Betania, San } \\
\text { Carlos }\end{array}$ & - & 3200 & Bmh-P/TB & Infiernito 3348 III \\
\hline Olman González & OG & $\begin{array}{l}\text { Betania, San } \\
\text { Carlos }\end{array}$ & - & 3200 & Bmh-P/TB & Tres Amigos 3347 I \\
\hline Carlos Vega & $\mathrm{CV}$ & $\begin{array}{l}\text { CoopeVega, San } \\
\text { Carlos }\end{array}$ & 88 & 3200 & Bmh-P/TB & Infiernito 3348 III \\
\hline Chimurria & $\mathrm{CH}$ & $\begin{array}{l}\text { Chimurria, } \\
\text { Upala }\end{array}$ & 85 & 2201 & $\mathrm{Bh}-\mathrm{T} / \mathrm{TP}$ & San Jorge 3248 II \\
\hline $\begin{array}{l}\text { Centro Agrícola } \\
\text { Cantonal Los } \\
\text { Chiles } 1\end{array}$ & $\mathrm{CACCH} 1$ & $\begin{array}{l}\text { Los Chiles, Los } \\
\text { Chiles }\end{array}$ & 212 & 2201 & Bh-T & Medio Queso 3248 I \\
\hline $\begin{array}{l}\text { Centro Agrícola } \\
\text { Cantonal Los } \\
\text { Chiles } 2\end{array}$ & $\mathrm{CACCH} 2$ & $\begin{array}{l}\text { Los Chiles, Los } \\
\text { Chiles }\end{array}$ & 212 & 2201 & Bh-T & Medio Queso 3248 I \\
\hline Luis F. Peña & ESC & $\begin{array}{l}\text { Escaleras, Los } \\
\text { Chiles }\end{array}$ & 70 & 2201 & Bh-T & Medio Queso 3248 I \\
\hline Guillermo Rojas & MA & $\begin{array}{l}\text { Montealegre, } \\
\text { Los Chiles }\end{array}$ & - & 2201 & Bh-T & Medio Queso 3248 I \\
\hline
\end{tabular}

(*)Información a partir de Delgado (2002). 
Los sitios incluidos en este estudio se dispersaron en un rango altitudinal comprendido entre los $70 \mathrm{msnm}$ y los $212 \mathrm{msnm}$, con rangos de precipitación que van desde los $2200 \mathrm{~mm}$, en las áreas con menor precipitación, hasta los 3200 $\mathrm{mm}$ en las zonas más lluviosas (Cuadro 1). Las zonas de vida son las siguientes: Bosque húmedoTropical (Bh-T), Bosque muy húmedo-Tropical (Bmh-T), Bosque húmedo-Tropical/Transición a Premontano (Bh-T/TP), Bosque muy húmedoPremontano/Transición a Basal (Bmh-P/TB).

\section{Diseño de muestreo y toma de la información de campo}

\section{Sistema de muestreo}

La selección de los sitios de estudio se realizó con la información ecológica y dasométrica de la plantación. Para el estudio dasométrico y epidométrico de la masa, se trabajó con datos provenientes de plantaciones que se encontraban bajo manejo forestal dentro de un proyecto administrado y ejecutado por COSEFORMA /Instituto Tecnológico de Costa Rica (TEC). Para el muestreo de los suelos realizado en los 12 sitios seleccionados se utilizaron parcelas temporales de 400 $\mathrm{m}^{2}$ que es un tamaño utilizado para disminuir la variación "dentro" de las parcelas y maximizar la variación "entre" ellas. Las parcelas fueron ubicadas de tal manera que las características del terreno y del arbolado representaran adecuadamente al resto de la plantación. Aún cuando las plantaciones de mayor edad alcanzaban los 12 años, la edad promedio de las plantaciones de $V$. guatemalensis, contempladas en el estudio, fue estimada en 9 años; y se consideró como la "edad base" para desarrollar el sistema de clasificación de sitios y asignar los valores de índice de sitio a cada una de las parcelas.

\section{Clasificación de la capacidad productiva de los terrenos mediante el índice de sitio}

Se utilizó el índice de sitio (IS), calculado a la edad base de 9 años, como la variable indicadora del potencial productivo de los sitios. Para construir las curvas de índice de sitio y asignar los valores correspondientes a las parcelas, se usó la siguiente ecuación ajustada en un trabajo previo (Mora y Meza, sin publicar), la cual está basada en el modelo de Bailey y Clutter (1974), donde LOG $($ Hdom $)=$ logaritmo de la altura dominante y $(1 / \mathrm{Edad})^{\hat{\mathrm{c}}}=$ el inverso de la edad elevado al tercer coeficiente; y a=5,74486, b=-5,19924 y $\hat{\mathrm{c}}=0,28782$, son los coeficientes del modelo.

$$
\operatorname{LOG}(\text { Hdom })=5,74486-5,19924 *(1 / \text { Edad })^{\wedge 0,28782}
$$

Los siguientes son los estadísticos que respaldan la función ajustada a los datos de la muestra: Coeficiente de Correlación r=-0,9133; Coeficiente de Determinación $\left(\mathrm{R}^{2}\right)=83,41$; Coeficiente de Determinación Ajustado $\left(\mathrm{R}^{2} \mathrm{adj}\right)=83,33$; Cuadrado Medio del Error (MSE)=0,0844; Error Estándar de las Estimaciones (SEE)=0,3266; Error Medio Absoluto (MAE)=0,2480.

El modelo de Bailey y Clutter (1974) también fue utilizado por Mora y Meza (2003) para construir las curvas de índice de sitio para
Tectona grandis en la Vertiente del Pacífico de Costa Rica.

\section{Muestreo y análisis de los suelos}

En este estudio de calidad de sitio, se utilizó el método de "profundidad predeterminada" para la toma de las muestras de suelo. Dentro de cada parcela temporal, se efectuó un muestreo sistemático del suelo, con muestras recolectadas a una distancia equidistante entre 
los puntos de muestreo con el fin de reducir su variabilidad. Las submuestras que se tomaron dentro de las 12 parcelas de $400 \mathrm{~m}^{2}$, fueron entremezcladas para obtener una muestra compuesta para cada parcela. Se procuró que dentro de cada parcela de muestreo las condiciones del micrositio fueran lo más homogéneas posibles. En cada una de las parcelas se tomaron muestras compuestas de suelo a 2 profundidades entre 0 y $20 \mathrm{~cm}$ y entre 20 y $40 \mathrm{~cm}$ para su posterior análisis en el laboratorio.

Las muestras de suelo así colectadas se analizaron en el Laboratorio de Análisis de Suelos y Foliares del Instituto de Investigación y Servicios Forestales (INISEFOR), de la Universidad Nacional. El análisis físico de suelos comprendió la determinación de los porcentajes de arena (Aren), arcilla (Arc) y limo (Limo) por el método del hidrómetro de Bouyoucos (Briceño y Pacheco 1984); densidad aparente (DAp) por el método del cilindro muestreador y retención de humedad determinada a $1 / 3$ de atmósfera (Hum1) y 15 atmósferas (Hum2) por las ollas de presión (Sampat 1979) mientras que los análisis químicos comprendieron $\mathrm{pH}$ en agua $(\mathrm{pH})$ en una relación suelo solución 1:2,5; acidez extraíble (AcExtr) con $\mathrm{KCl} 1 \mathrm{M}$ y determinada por valoración con $\mathrm{NaOH}$ (Jackson 1964); fósforo (P) extraído con solución extractora Olsen Modificada $\mathrm{pH}$ 8,5 y determinado por colorimetría; Ca y Mg extraídos con $\mathrm{KCl} 1 \mathrm{M}$ y determinados por espectrofotometría de absorción atómica; $\mathrm{K}, \mathrm{Fe}, \mathrm{Cu}, \mathrm{Mn}$ y Zn, extraídos con solución extractora Olsen Modificada $\mathrm{pH} 8,5$ y determinados por espectrofotometría de absorción atómica y materia orgánica (MO) determinada por oxidación en medio ácido con dicromato de potasio (Díaz Romeo y Hunter 1982). Además, al relacionar las variables ya descritas se pudo determinar, mediante cálculos aritméticos las siguientes variables derivadas: suma de bases ( $\Sigma$ Bases), capacidad de intercambio de cationes efectiva (CICE), saturación de bases (SatBa), saturación de acidez (SatAc), saturación de calcio (SatCa), saturación de magnesio (SatMg), saturación de potasio (SatK), relación calcio/magnesio $(\mathrm{Ca} /$ $\mathrm{Mg}$ ), calcio/potasio $(\mathrm{Ca} / \mathrm{K})$, calcio+magnesio/ potasio $(\mathrm{Ca}+\mathrm{Mg} / \mathrm{K})$.

La interpretación de los resultados obtenidos en los sitios estudiados y la valoración química de los suelos para las 2 profundidades, se hizo con las normas establecidas en el "Manual para interpretar la fertilidad de suelos" (Berstch 1986).

\section{Características de los órdenes de suelo presentes en la región estudiada}

En la región bajo estudio se encontraron 2 órdenes de suelo. Por un lado los Ultisoles, suelos muy intemperizados de baja fertilidad natural que se localizan en posiciones geomorfológicas viejas. Presentan alta acidez, debido a la pérdida de bases por lixiviación lo cual incide en su baja productividad. Según Kamprath (1967) y Woodruff y Kamprath (1965) la mayoría de estos suelos se caracterizan fundamentalmente por tener deficiencias de Ca y $\mathrm{Mg}$, toxicidad de $\mathrm{Fe}, \mathrm{Al}$ y $\mathrm{Mn}$, baja actividad de microorganismos y disminución en la disponibilidad de fósforo. Sus características principales son la presencia de un horizonte argílico en el perfil y una baja saturación de bases menor de un 35\%. El horizonte argílico es un horizonte subsuperficial de naturaleza iluvial o sea de enriquecimiento con arcilla, debido a la migración mecánica de la arcilla desde un horizonte superior (eluvial) y es por consiguiente un horizonte de acumulación de arcilla iluvial. Para este orden la precipitación es uno de los factores de formación de suelos más importante puesto que favorece la translocación del material de una parte del perfil a zonas inferiores por procesos de eluviación e iluviación, que influyen en el desarrollo del perfil del suelo (Buol et ál. 1980).

Por otra parte los Inceptisoles son suelos incipientes que manifiestan ciertas evidencias, aunque débiles en su evolución edáfica, pero que empiezan a mostrar el desarrollo de los horizontes puesto que son aun recientes suelos en evolución. Es por ello que dentro de este orden, aparecen suelos con uno o más horizontes de 
diagnóstico, cuya génesis es de formación rápida, con procesos de translocación de materiales. Entre ellos podemos encontrar desde suelos mal drenados, hasta suelos muy bien drenados en todo tipo de pendientes (CATIE 1984).
En el Cuadro 2 se presenta la distribución de las clases de calidad de sitio en relación con los órdenes de suelo mencionados; además, se agregan algunas de las características más relevantes de los mismos.

Cuadro 2. Clases de fertilidad de 0 a $20 \mathrm{~cm}$ y clasificación taxonómica por categorías de calidad de sitio para 12 localidades plantadas con V. guatemalensis, Región Huetar Norte de Costa Rica.

\begin{tabular}{|c|c|c|c|c|c|}
\hline Clase de sitio & Localidades & $\begin{array}{l}\text { IS } \\
\text { Prom }\end{array}$ & $\begin{array}{l}\text { Fertilidad } \\
(\text { de } 0 \text { a } 20 \mathrm{~cm})\end{array}$ & $\begin{array}{l}\text { Orden de } \\
\text { Suelo }\end{array}$ & Grandes grupos de suelc \\
\hline Excelente & $\begin{array}{l}\mathrm{LT} \\
\mathrm{OR} 2\end{array}$ & 25,08 & Baja & Ultisol & $\begin{array}{l}\text { Tropohumult } \\
\text { Tropohumult }\end{array}$ \\
\hline Muy bueno & $\begin{array}{l}\text { OR4 } \\
\text { OG } \\
\mathrm{MV} \\
\mathrm{CV} \\
\mathrm{CH}\end{array}$ & 23,09 & Baja & Ultisol & $\begin{array}{l}\text { Tropohumult } \\
\text { Tropohumult } \\
\text { Tropohumult } \\
\text { Tropohumult } \\
\text { Tropohumult }\end{array}$ \\
\hline Regular & $\begin{array}{l}\text { ER } \\
\text { CACCH2 } \\
\text { ESC }\end{array}$ & 18,85 & Media & Inceptisol & $\begin{array}{l}\text { Tropohumult } \\
\text { Dystropept } \\
\text { Dystropept }\end{array}$ \\
\hline Malo & $\begin{array}{l}\text { MA } \\
\text { CACCH1 }\end{array}$ & 14,99 & Media & Inceptisol & $\begin{array}{l}\text { Dystropept } \\
\text { Dystropept }\end{array}$ \\
\hline
\end{tabular}

Determinación de los factores críticos para el crecimiento de $V$. guatemalensis en la Región Huetar Norte de Costa Rica

Para identificar los factores edáficos críticos que determinan el crecimiento de $V$. guatemalensis, se procedió a trabajar con una muestra de suelos de 0 a $20 \mathrm{~cm}$ (Cuadro 3) y otra de 20 a 40 $\mathrm{cm}$ (Cuadro 4), con un total 23 variables edáficas (variables independientes) para cada profundidad de suelo y el índice de sitio (IS) como variable de clasificación o variable dependiente. Los datos presentes en los cuadros mencionados, se ordenaron en forma decreciente de acuerdo con el índice de sitio de manera que los mejores sitios (sitios de mayor calidad) quedaran en la parte superior de los mismos y los peores sitios (sitios de mala calidad) ocuparan las posiciones inferiores; esto con el fin de mostrar la distribución de los mismos y de contrastar las diferencias entre los mejores y los peores sitios para la especie. Para mantener la claridad y mejorar la comprensión en la discusión de los resultados se utilizaron los promedios por clase de sitio de las variables mejor relacionadas con la variable de clasificación (IS).

Luego se procedió a examinar los archivos a través de variables edáficas en las que se pudiera identificar algún tipo de relación con el índice de sitio. Esto permitió identificar aquellas variables que mostraron una secuencia clara y un patrón definido con respecto a la variable dependiente; todas las demás variables fueron excluidas 


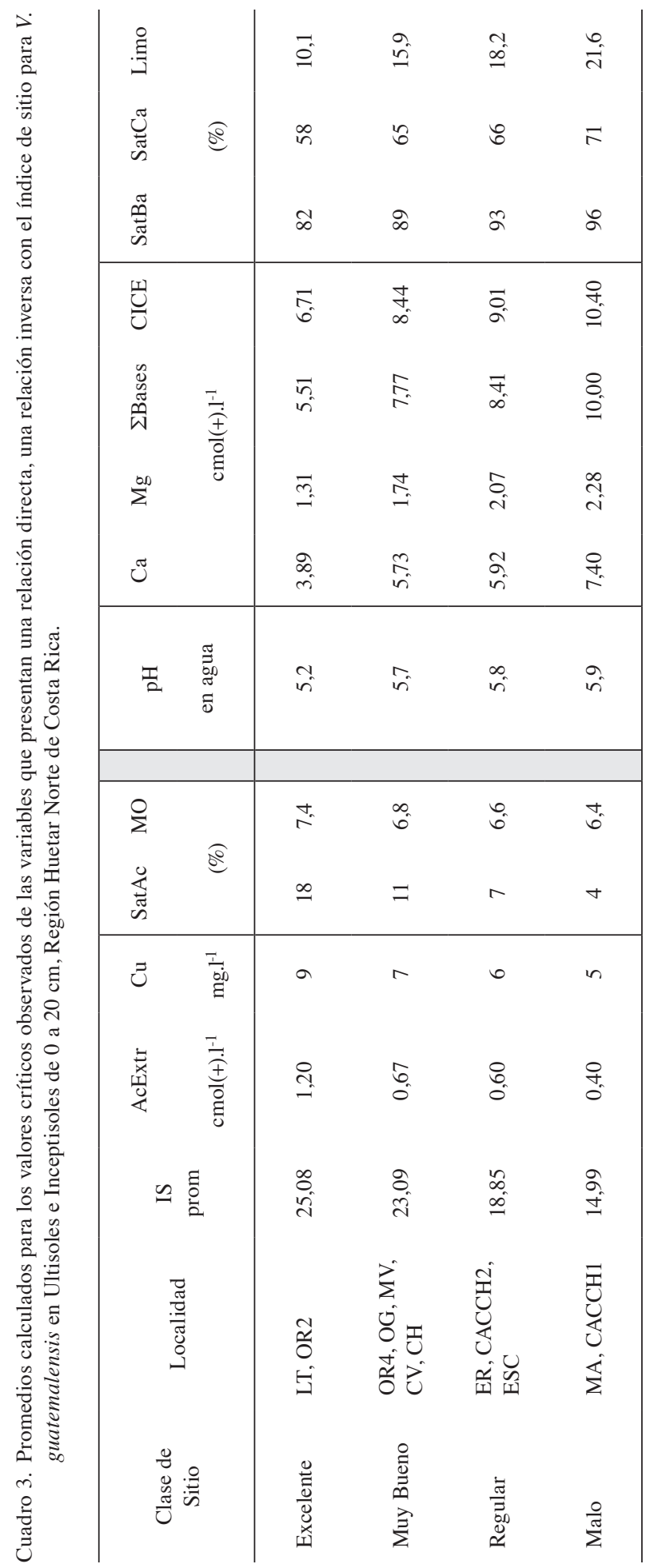


Cuadro 4. Promedios calculados para los valores críticos observados de las variables que presentan una relación directa, o una relación inversa con el índice de sitio para V. guatemalensis en Ultisoles e Inceptisoles de 20 a 40 cm, Región Huetar Norte de Costa Rica.

\begin{tabular}{|c|c|c|c|c|c|c|c|c|c|}
\hline $\begin{array}{l}\text { Clase de } \\
\text { sitio }\end{array}$ & Localidades & $\begin{array}{l}\text { IS } \\
\text { prom }\end{array}$ & $\begin{array}{c}\text { DAp } \\
\left(\mathrm{g} . \mathrm{cm}^{-3}\right)\end{array}$ & $\begin{array}{l}\text { Arc } \\
(\%)\end{array}$ & $\begin{array}{c}\mathrm{Fe} \\
\mathrm{mg} \cdot \mathrm{l}^{-1}\end{array}$ & $\mathrm{Ca} / \mathrm{K}$ & $(\mathrm{Ca}+\mathrm{Mg}) / \mathrm{K}$ & $\mathrm{MO}$ & $\begin{array}{l}\text { Limo } \\
\%)\end{array}$ \\
\hline Excelente & LT, OR2 & 25,08 & 1,14 & 65,90 & 166 & 23,8 & 32,1 & 3,4 & 14,80 \\
\hline Muy Bueno & $\begin{array}{l}\mathrm{OR} 4, \mathrm{OG}, \mathrm{MV}, \\
\mathrm{CV}, \mathrm{CH}\end{array}$ & 23,09 & 1,16 & 72,24 & 110 & 15,4 & 20,6 & 3,2 & 12,44 \\
\hline Regular & $\begin{array}{l}\text { ER, CACH2, } \\
\text { ESC }\end{array}$ & 18,85 & 1,34 & 81,20 & 100 & 14,8 & 20,5 & 3,1 & 9,73 \\
\hline Malo & $\mathrm{MA}, \mathrm{CACH} 1$ & 14,99 & 1,36 & 82,30 & 68 & 13,3 & 17,6 & 2,7 & 8,30 \\
\hline
\end{tabular}

del análisis. Por otra parte, con las variables que sobrevivieron a este primer escrutinio, se procedió a separar para cada profundidad, las variables que mostraron una "relación directa" con el índice de sitio (relación positiva) y las variables que presentaron una "relación inversa" con la variable dependiente (relación negativa).

Para la construcción de los Cuadros 3 y 4, se conformaron 5 clases de calidad de sitio de acuerdo con la clasificación de sitios para V. guatemalensis (Mora y Meza sin publicar); para, 1. sitios excelentes (IS=mayores a 25,08); 2. sitios muy buenos (IS=de 23,09 a 25,07); 3 . sitios buenos (IS = de 20,97 a 23,08); 4. sitios regulares (IS=de 18,85 a 20,96); y, 5. sitios malos (IS=menores de 18,84); todos los IS están referidos a la edad de 9 años.

Las clases mencionadas permitieron identificar los valores extremos para la variable dependiente (IS) y los correspondientes valores de las variables asociadas. De esta manera, los valores de las variables edáficas ubicados en la parte inferior de los cuadros asociados a los índices de sitio más bajos se consideraron como factores limitantes para el crecimiento de la especie y los valores ubicados en la parte superior de los cuadros asociados a los índices de sitio más altos fueron asumidos como condiciones favorables para el crecimiento de $V$. guatemalensis, independientemente del tipo de relación (directa o indirecta, positiva o negativa) que mostraran con el índice de sitio.

\section{Análisis multivariado de las variables mejor correlacionadas con el índice de sitio}

Después de determinar los factores críticos para el crecimiento de $V$. guatemalensis se realizó un análisis multivariado con las variables del suelo seleccionadas en el proceso anterior por medio del programa estadístico InfoStat (versión 2011e), a través del procedimiento Biplot el cual es una técnica para la representación simultánea de observaciones y de variables (Balzarini et ál. 2008) de tal forma que sobre el mismo plano se superponen $p$ puntos que representan todas las variables (Tusell 2012) lo cual hace interpretables las relaciones entre ellas y las observaciones (Balzarini et ál. 2008). De acuerdo con Tusell (2012) la interpretación de un Biplot, va a depender del ángulo que se forma entre los vectores y por consiguiente, si una variable $j$ tiene gran influencia en la observación $i$, los vectores que representan a ambas, tenderán a formar un ángulo pequeño. 


\section{RESULTADOS Y DISCUSIÓN}

\section{Relación entre los índices de sitio para $V$. guatemalensis y las clases de fertilidad por órdenes de suelo}

Se observa que los mejores sitios para el crecimiento de $V$. guatemalensis se ubican en suelos Ultisoles (Cuadro 2) ya que las clases excelente y muy bueno están asociadas con las condiciones que presentan estos suelos, esto es, baja fertilidad, $\mathrm{pH}$ bajos, alta acidez y altos contenidos de aluminio intercambiable (Piotto et ál. 2010). Aunque los Ultisoles son de baja fertilidad, tienen buenas propiedades físicas asociadas con una buena estructura, lo que permite un mejor crecimiento de la especie, desde el punto de vista hídrico y de absorción de nutrimentos. Por el contrario, las calidades regular y malo se ubican en suelos Inceptisoles que son de mediana fertilidad.

Comparados con los Ultisoles, los Inceptisoles carecen de buenas condiciones físicas; lo cual puede verse reflejado en el crecimiento de la especie, ya que puede provocar que exista una menor proporción de raíces superficiales y en profundidad, lo que le impide explorar un mayor volumen de suelo en el perfil. No obstante, esta afirmación debe ser investigada más a fondo, ya que Piotto et ál. (2010) mencionan, entre otras cosas, que la especie es capaz de soportar suelos de mal drenaje, y en tal caso, este no sería un factor determinante para un mal desempeño.

En la Figura 1 se presenta la variación de los suelos y la distribución de los sitios de muestreo en la región bajo estudio. Se puede observar que de los 5 grandes grupos representados en la zona, solamente en 2 de ellos se ubican puntos de muestreo; estos son, Tropohumult, que pertenece al orden de los Ultisoles y donde se localiza la mayor parte de los sitios estudiados (un total de 8), y Dystropept, el cual pertenece al orden de los Inceptisoles (con 4 sitios de estudio). Cabe mencionar que estos 2 grandes grupos ocupan las áreas de mayor extensión en dicha zona.

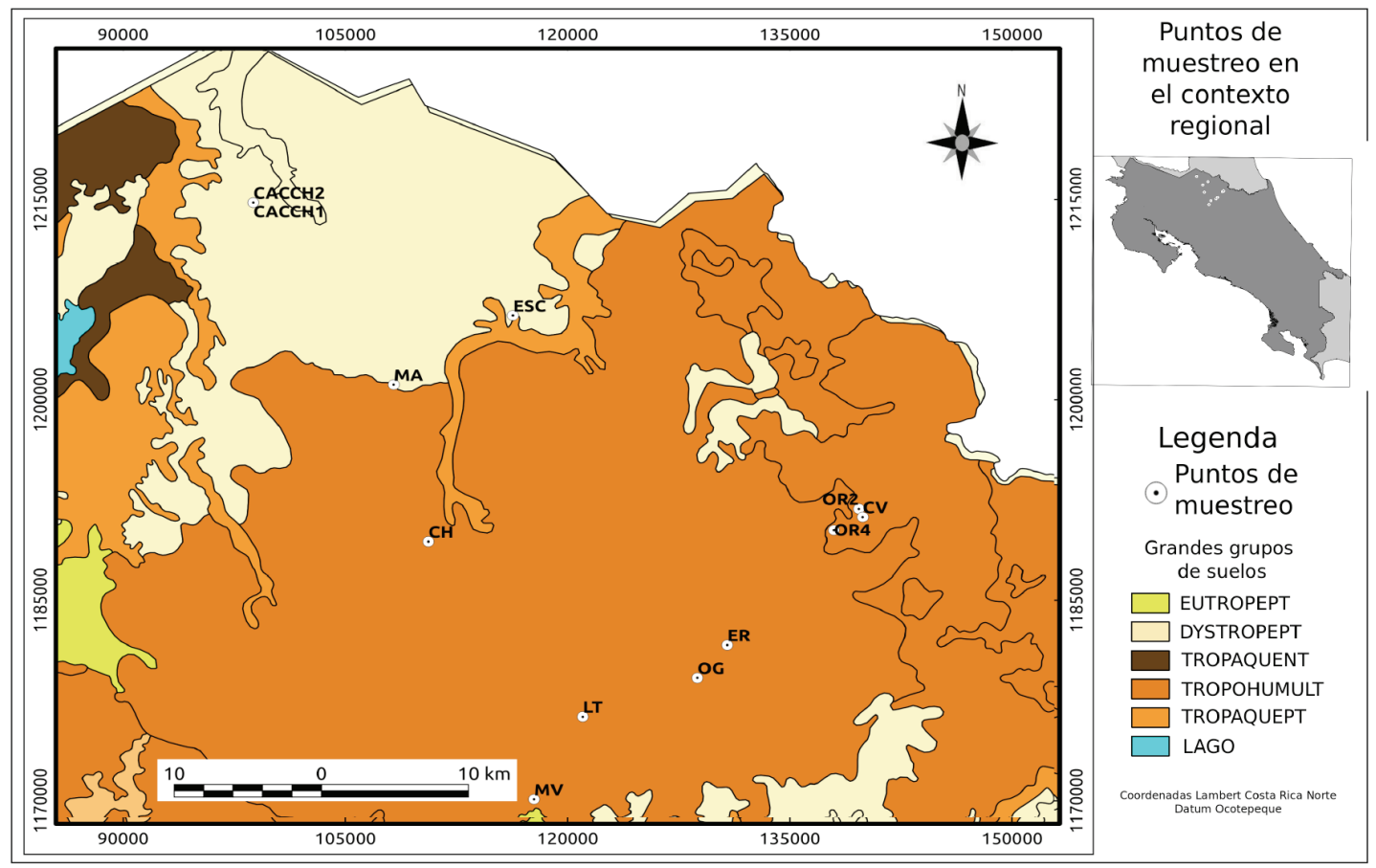

Fig. 1. Mapa de la región estudiada que muestra la variación de los suelos y la distribución de los puntos de muestreo en plantaciones de Vochysia guatemalensis, Región Huetar Norte de Costa Rica. 
Análisis de los valores críticos para el crecimiento y la producción de $V$. guatemalensis observados a una profundidad de 0 a $20 \mathrm{~cm}$

Las variables edáficas que mejor se relacionan con el índice de sitio (ya sea directa o inversamente), denominados valores críticos observados para el crecimiento y la producción de $V$. guatemalensis, a una profundidad de 0 a 20 $\mathrm{cm}$, se presentan en el Cuadro 3. Aquí se observa la correspondencia que existe entre los valores extremos de las variables del suelo, con los valores del índice de sitio (IS) definidos a la edad de 9 años. Así, los valores más bajos de las variables del suelo que mantienen una relación inversa con el índice de sitio (pH, Ca, Mg, $\Sigma$ Bases, CICE, SatBa, SatCa, Limo), corresponden a las localidades ubicadas en la clase excelente (LT y OR2; ISprom=25,08), esto es, la clase con el índice de sitio más alto; mientras que los valores más altos de las mismas corresponden a las localidades que presentan el índice de sitio más bajo (MA y CACCH1; ISprom=14,99); o sea, la peor clase de sitio. Por otra parte, los valores más altos de las variables del suelo que mantienen una relación directa con el índice de sitio (AcExtr, Cu, SatAc, MO), se asocian con la clase de sitio más alta, que corresponde a las localidades que presentan el índice de sitio más alto, esto es sitios excelentes; mientras que los valores más bajos de las mismas corresponden a las localidades que presentan el índice de sitio más bajo, o sea, a la clase de sitio mala.

Con este tipo de relaciones se puede identificar, por un lado, los factores limitantes, y por otro lado, las condiciones más favorables para el crecimiento de esta especie, en la Región Huetar Norte de Costa Rica.

\section{Factores limitantes para el crecimiento y la producción de $V$. guatemalensis a una profundidad de 0 a $20 \mathrm{~cm}$}

De acuerdo con los datos colectados, de 0 a $20 \mathrm{~cm}$, los peores sitios parecen estar relacionados con un $\mathrm{pH}$ medio $(5,9)$, concentraciones medias de $\mathrm{Ca}\left(7,40 \mathrm{cmol}(+) . \mathrm{l}^{-1}\right)$ y $\mathrm{Mg}\left(2,28 \mathrm{cmol}(+) .1^{-1}\right)$, una $\Sigma$ Bases $\left(10,0 \mathrm{cmol}(+) .1^{-1}\right)$ y una CICE $(10,40$ $\left.\mathrm{cmol}(+) .1^{-1}\right)$ medias, una SatBa $(96 \%)$ y una SatCa (71\%) altas, una $\operatorname{AcExtr}\left(0,40 \mathrm{cmol}(+) \mathrm{l}^{-1}\right)$ baja, concentraciones medias de $\mathrm{Cu}\left(5 \mathrm{mg} . \mathrm{l}^{-1}\right)$, una SatAc baja (4\%) y de acuerdo con Hardy, citado por Fassbender (1975), un contenido de MO alto $(6,4 \%)$. Estos valores se podrían considerar como indicadores de las condiciones más desfavorables para el crecimiento y la producción de esta especie, en la Región Huetar Norte de Costa Rica, a una profundidad de 0 a $20 \mathrm{~cm}$.

Se observa el porcentaje de saturación de acidez, es notorio ya que $V$. guatemalensis crece menos en aquellos sitios con valores $<11 \%$, contrario a lo que presenta Tectona grandis, que mostró los mejores crecimientos en sitios con valores de saturación de acidez $<8 \%$ (Mollinedo et ál. 2005). Por su parte, Alvarado y Fallas (2004) determinaron que valores mayores a un $3 \%$ en la saturación de acidez del suelo reducen, significativamente, el incremento medio anual en altura en plantaciones de Teca menores de 5 años.

\section{Condiciones favorables para el crecimiento y la producción de $V$. guatemalensis a una profundidad de 0 a $20 \mathrm{~cm}$}

Los mejores sitios parecen estar relacionados con pH's bajos $(5,2)$, bajas concentraciones de $\mathrm{Ca}\left(3,89 \mathrm{cmol}(+) \cdot 1^{-1}\right)$ y concentraciones medias de $\operatorname{Mg}\left(1,31 \mathrm{cmol}(+) .1^{-1}\right)$, $\Sigma$ Bases $\left(5,51 \mathrm{cmol}(+) .1^{-1}\right)$ y una CICE $\left(6,71 \mathrm{cmol}(+) .1^{-1}\right)$ medias, una SatBa $(82 \%)$ y una SatCa $(58 \%)$ medias, una AcExtr $\left(1,20 \mathrm{cmol}(+) . \mathrm{l}^{-1}\right)$ media, con concentraciones medias de $\mathrm{Cu}\left(9 \mathrm{mg} \cdot \mathrm{l}^{-1}\right)$, con una SatAc media (18\%) y según Hardy, citado por Fassbender (1975), un alto contenido de materia orgánica $(7,4 \%)$. Estos valores se podrían considerar como identificadores de las condiciones más favorables para el crecimiento y la producción de $V$. guatemalensis, en la Región Huetar Norte de Costa Rica, de 0 a $20 \mathrm{~cm}$ de profundidad.

Los resultados parecen resaltar los bajos requerimientos nutricionales que presenta $V$. guatemalensis (Cuadros 3) para alcanzar un índice de sitio promedio de 25,08 a la edad de 9 años. Un $\mathrm{pH}$ 
más bajo, menores concentraciones de $\mathrm{Ca}$ y $\mathrm{Mg}$, una menor CICE y una menor suma de bases, una mayor acidez y un mayor porcentaje de saturación de acidez en los mejores sitios, sugieren que $V$. guatemalensis tiene la capacidad de crecer y de ser más productiva bajo tales condiciones. El crecimiento de la especie no se ve afectado en suelos relativamente ácidos. De la misma manera, Herrera y Campos (1999) encontraron para $V$. ferruginea, una mayor productividad en sitios con un pH ácido, alta saturación de acidez, altas concentraciones de $\mathrm{Fe}$ y $\mathrm{Al}$ y bajas concentraciones de bases intercambiables.

\section{Análisis de los valores críticos para el crecimiento y la producción de $V$. guatemalensis observados a una profundidad de 20 a $40 \mathrm{~cm}$}

Los valores críticos observados para el crecimiento y la producción de $V$. guatemalensis (variables edáficas que mejor se relacionan con el IS, directa o inversamente), observados en la región bajo estudio, a una profundidad de 20 a $40 \mathrm{~cm}$, se presentan en el Cuadro 4. En este se comprueba que existe una correspondencia entre los valores extremos de las variables del suelo con los valores del índice de sitio (IS) definidos a la edad de 9 años. Los valores más bajos de las variables del suelo que se relacionan de manera contrapuesta (relación inversa) con el IS (DAp y Arc), se encuentran asociados con los mejores sitios (LT y OR2, ISprom $=25,08$ ), mientras que los valores más altos de las mismas corresponden a los sitios más malos registrados en la muestra (MA y CACCH1, ISprom=14,99). Por otra parte, los valores más altos de las variables que muestran una relación directa con el índice de sitio (Fe, $\mathrm{Ca} / \mathrm{K},(\mathrm{Ca}+\mathrm{Mg}) / \mathrm{K}, \mathrm{MO}$ y Limo), se asocian con los sitios excelentes y los valores más bajos se asocian con los sitios malos.

\section{Factores limitantes para el crecimiento y la producción de $V$.guatemalensis a una profundidad de 20 a $40 \mathrm{~cm}$}

De acuerdo con los resultados obtenidos por la muestra, de 20 a $40 \mathrm{~cm}$, las peores condiciones para el crecimiento de esta especie están asociadas con una DAp media $\left(1,36 \mathrm{~g} . \mathrm{cm}^{-3}\right)$, un suelo muy arcilloso $(82,30 \%)$, así como un contenido medio de $\mathrm{Fe}\left(68 \mathrm{mg} . \mathrm{l}^{-1}\right)$, una relación $\mathrm{Ca} / \mathrm{K}$ adecuada $(13,3)$ y una relación $\mathrm{Ca}+\mathrm{Mg} / \mathrm{K}$ también adecuada $(17,6)$, y de acuerdo con Hardy (citado por Fassbender 1975), una proporción de MO media (2,7\%). Estos valores se podrían considerar como indicadores de las condiciones más desfavorables para el crecimiento y la producción de $V$. guatemalensis, en la Región Huetar Norte de Costa Rica, entre $20 \mathrm{~cm}$ y $40 \mathrm{~cm}$ de profundidad.

Se observa menor crecimiento de $V$. guatemalensis, entre los 20 y los $40 \mathrm{~cm}$, en los sitios que presentan mayores valores de densidad aparente conforme aumenta la profundidad. En el perfil del suelo, la densidad aparente tiende a aumentar con la profundidad, debido a una disminución en los contenidos de materia orgánica, lo cual influye en una menor agregación de las partículas del suelo y una menor porosidad total en los horizontes más profundos, los cuales, por lo general, son más compactos que los superficiales. Por otra parte, con la profundidad aumenta la compactación causada por el peso de las capas superiores sobre las capas subsuperficiales, lo que aumenta la acumulación de materiales sólidos en los macroporos, y disminuye el volumen de poros. Al incrementar los valores de densidad aparente, la resistencia mecánica tiende a aumentar y la porosidad del suelo tiende a disminuir, el efecto se manifiesta como una limitación en el crecimiento de las raíces a valores críticos. Estos valores críticos para el crecimiento de las raíces varían, según la textura del suelo y la especie forestal, y en el caso de V. guatemalensis han sido estudiados por Moreira y Arnáez (2007).

Como lo muestra el Cuadro 4, hay un incremento en los contenidos de arcilla en la composición textural de los sitios que presentan un menor crecimiento de la especie, a una profundidad de 20 a $40 \mathrm{~cm}$; estos altos porcentajes de arcilla están asociados con un proceso de compactación que ocurre conforme aumenta la profundidad del suelo, lo cual disminuye el espacio 
poroso. Esto causa condiciones inadecuadas para el buen desarrollo de los árboles, que afectan la distribución de las raíces al disminuir su crecimiento e impedir su penetración, aspecto que representa una limitación en la profundidad efectiva del suelo, además, se afecta la difusión del aire en el mismo así como el movimiento del agua.

\section{Condiciones favorables para el crecimiento y la producción de $V$.guatemalensis a una profundidad de 20 a $40 \mathrm{~cm}$}

Por otra parte, los mejores sitios parecen estar relacionados con una baja DAp $\left(1,14 \mathrm{~g} . \mathrm{cm}^{-3}\right)$, un suelo arcilloso $(65,90 \%)$, un alto contenido de $\mathrm{Fe}$ (166 mg. $\mathrm{l}^{-1}$ ), una relación $\mathrm{Ca} / \mathrm{K}$ adecuada $(23,8)$, una relación $\mathrm{Ca}+\mathrm{Mg} / \mathrm{K}$ también adecuada $(32,1)$ y según Hardy (citado por Fassbender 1975), una cantidad media de MO (3,4\%). Estos últimos valores se podrían considerar como identificadores de las condiciones más favorables para el crecimiento y la producción de $V$. guatemalensis en la Región Huetar Norte de Costa Rica, a una profundidad de 20 a $40 \mathrm{~cm}$.

Se observa un mejor desarrollo de $V$. guatemalensis en los sitios con menores valores de densidad aparente, entre los 20 y los $40 \mathrm{~cm}$ de profundidad. La relación entre la materia orgánica y la densidad aparente constituye un buen indicador de la calidad de los suelos. Un incremento en los contenidos de materia orgánica promueve la agregación de las partículas minerales del suelo e incrementa el volumen total de poros, que a su vez provoca una disminución en la densidad aparente, debido al efecto mejorador de la materia orgánica en la estructura del suelo. Al aumentar el espacio poroso, es indudable que se dan mejores condiciones para el desarrollo vegetal; también se favorece el intercambio suelo-planta, la difusión del aire en el suelo y el movimiento del agua. Por lo tanto, con densidades aparentes menores, se crearían mejores condiciones físicas para las especies forestales.

Las altas concentraciones de Fe (mayores de $100 \mathrm{mg} . \mathrm{l}^{-1}$ ) en estos sitios, pueden estar asociadas a condiciones de suelos altamente meteorizados (caso de los Ultisoles), caracterizados por tener en su mineralogía, caolinita y óxidos e hidróxidos de hierro y aluminio. Altas concentraciones de $\mathrm{Fe}$ y Al, como las mostradas por los sitios excelentes y muy buenos (Cuadro 4), están relacionadas con condiciones de acidez en los suelos, lo cual sugiere que $V$. guatemalensis muestra bajos requerimientos nutricionales, debido a su capacidad para crecer en condiciones de alta acidez del suelo. Con respecto a la respuesta en el crecimiento, que presenta esta especie, a las condiciones de acidez asociadas al Al, Haridasan et ál. (1986), Haridasan y Monteiro (1988), Pérez et ál. (1993), mencionan que las especies del género Vochysia, tienen la capacidad de acumular el $\mathrm{Al}$ en diferentes estructuras de las hojas. Flores (1993) afirma que tanto $V$. ferruginea como $V$. guatemalensis acumulan más de $10.000 \mathrm{mg} \cdot \mathrm{kg}^{-1}$ de $\mathrm{Al}$ en los tejidos foliares, mientras que González y Fisher 1997 determinaron un contenido de $20.100 \mathrm{mg} \cdot \mathrm{kg}^{-1}$ de aluminio en las hojas; por su parte, Badilla (2012) encontró una respuesta similar en el tejido radical. Sin embargo, aun no se conocen los mecanismos fisiológicos involucrados en la adaptación de las especies de este género a los suelos ácidos.

Los resultados presentados en este documento sugieren que esta especie es menos productiva en suelos con concentraciones medias de hierro $\left(\geq 10<100 \mathrm{mg} \cdot \mathrm{l}^{-1}\right)$, lo cual confirma la capacidad de $V$.guatemalensis de ser más productiva en los sitios asociados con una alta acidez y altas concentraciones de Fe.

Los resultados del análisis estadístico realizado con el procedimiento Biplot que es una técnica para la representación simultánea de observaciones y variables se presentan en las Figuras 2 y 3. Los ángulos que se observan entre las líneas representan la relación que existe entre las variables y los ángulos pequeños que se forman, señalan que las variables analizadas tienen gran influencia sobre las observaciones y por el contrario, los ángulos grandes muestran que la influencia es reducida. Se observa la correspondencia entre las diferentes clases de calidad de sitio y las variables edáficas que mostraron una relación positiva, 


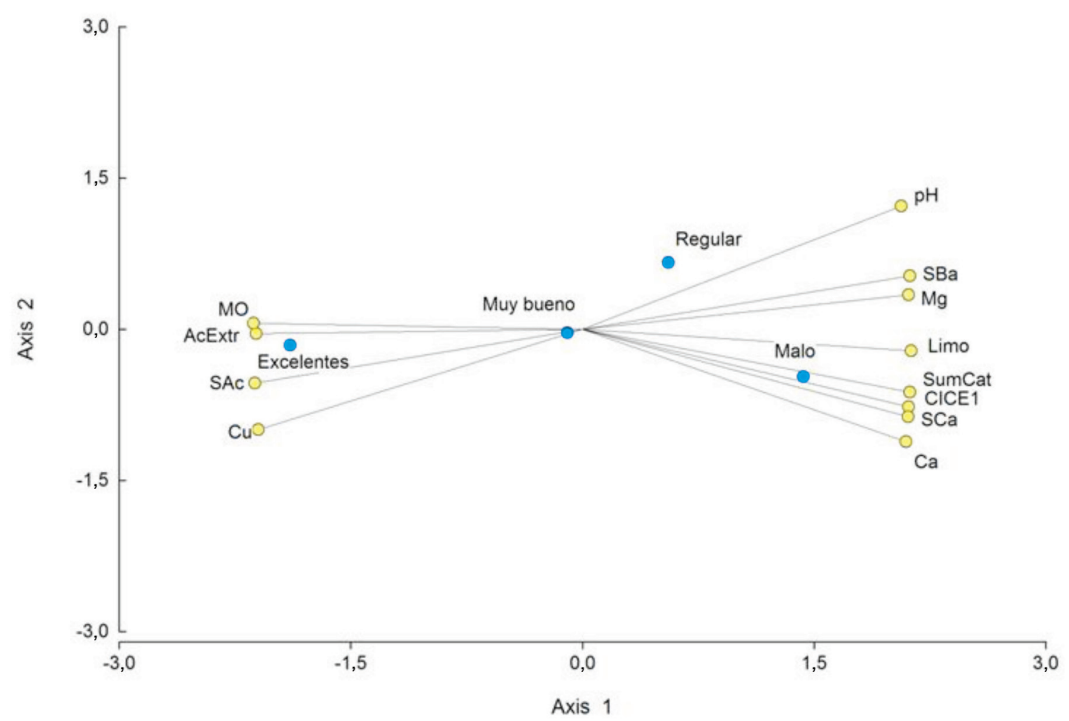

Fig. 2. Correspondencia entre la calidad de sitio y las variables edáficas relacionada directa o inversamente con el índice de sitio para V. guatemalensis, de 0 a 20 cm, Región Huetar Norte, Costa Rica.

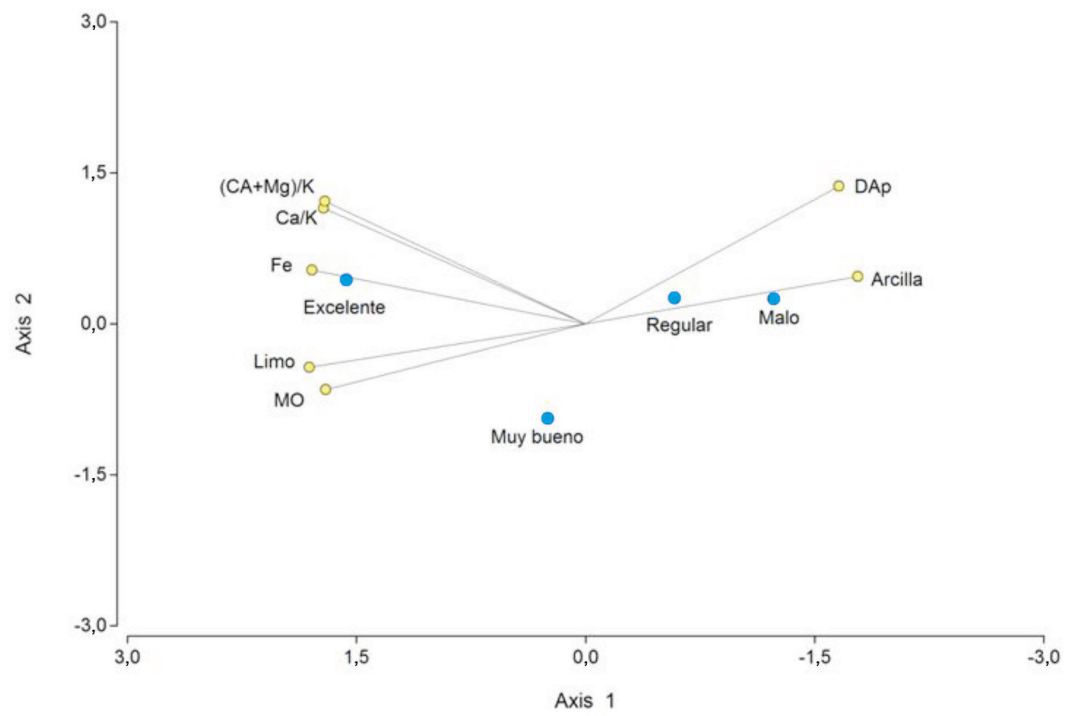

Fig. 3. Correspondencia entre la calidad de sitio y las variables edáficas relacionada directa o inversamente con el índice de sitio para V. guatemalensis de 20 a 40 cm, Región Huetar Norte, Costa Rica.

o bien, una relación negativa, con el índice de sitio para ambas profundidades (de 0 a $20 \mathrm{~cm}$ y de 20 a $40 \mathrm{~cm}$ ). En ambos casos se da un agrupamiento entre las 2 mejores calidades de sitio entre excelente y muy bueno y otro entre las de menor calidad como regular y malo. En el caso de la profundidad de 0 a $20 \mathrm{~cm}$ (Figura 2) las variables que se grafican en una misma dirección MO, 
AcExtr, SatAc y $\mathrm{Cu}$ tienen los valores más altos y se encuentran asociadas a las mejores calidades de sitio (relación positiva). En consecuencia, los valores bajos de estas variables se grafican en la dirección opuesta y están relacionados con los sitios de menor calidad. Por el contrario, los valores más altos de $\mathrm{pH}, \mathrm{Ca}, \mathrm{Mg}$, SumCat, CICE, SatBa, SatCa y Limo se relacionan con las condiciones de menor calidad. Por tanto, los valores menores de estas variables se asocian con los sitios de mejor calidad (relación negativa).

En la profundidad de 20 a $40 \mathrm{~cm}$ (Figura 3), los valores más altos de $\mathrm{Fe}, \mathrm{Ca} / \mathrm{K},(\mathrm{Ca}+\mathrm{Mg}) / \mathrm{K}$, MO y Limo son graficados en una misma dirección y son asociados a las mejores condiciones de sitio (relación positiva); inversamente, los valores más bajos de estas variables se asocian a las calidades de sitio menores. Por otra parte, DAp y Arc, siguen una dirección opuesta a la de las variables anteriores (relación negativa). Por lo tanto, los valores más bajos de estas variables están relacionados con los sitios de mejor calidad y los valores más altos de las mismas se asocian con los sitios de peor calidad. Por otra parte, MO es la única variable que repite en las 2 profundidades (Figuras 2 y 3 ), donde los valores más altos están asociados con las mejores calidades de sitio, en ambos casos.

\section{CONCLUSIONES}

En la muestra se encuentran representados un amplio intervalo de calidades de sitio y de condiciones edáficas, incluyendo valores que se pueden considerar extremos, lo que permitió identificar los factores críticos que influyen en el crecimiento de $V$. guatemalensis.

Los resultados obtenidos de 0 a $20 \mathrm{~cm}$ muestran que no existe una relación directa entre la composición química de los suelos y la calidad de sitio para V. guatemalensis. Para los sitios estudiados, se observa cómo los suelos que presentan una mejor condición química (mayores contenidos de $\mathrm{Ca}$ y $\mathrm{Mg}$, mayor suma de bases y saturación de bases, mayor CICE y menores valores de acidez extraíble y de saturación de acidez), son los que presentan la peor calidad de sitio para V. guatemalensis (IS menor de 20).

Los sitios menos favorables que comprenden de 0 a $20 \mathrm{~cm}$, están relacionados con un $\mathrm{pH}$ medio $(5,9)$, concentraciones medias de $\mathrm{Ca}\left(7,40 \mathrm{cmol}(+) \cdot 1^{-1}\right)$ y $\mathrm{Mg}\left(2,28 \mathrm{cmol}(+) \cdot 1^{-1}\right)$, una $\Sigma$ Bases $\left(10,0 \mathrm{cmol}(+) .^{-1}\right)$ y una CICE $(10,40$ $\left.\mathrm{cmol}(+) \cdot \mathrm{l}^{-1}\right)$ medias, una Ac.Extr. $\left(0,40 \mathrm{cmol}(+) .1^{-1}\right)$ y una SatAc baja (4\%). Al analizar los resultados obtenidos de 20 a $40 \mathrm{~cm}$ se considera que las condiciones menos favorables para el crecimiento de esta especie están asociadas con una DAp media $\left(13,6 \mathrm{~g} \mathrm{~cm}^{-3}\right)$, un suelo muy arcilloso Arc $(82,30 \%)$, así como un contenido medio de $\mathrm{Fe}$ (68 mg. $\left.1^{-1}\right)$ y con poca $\mathrm{MO}(2,7 \%)$.

Para los sitios estudiados, se observa que a estas 2 profundidades los suelos presentan mejor condición química, de acuerdo con el "Manual para interpretar la fertilidad de suelos" (Berstch 1986), son los que presentan las peores condiciones de sitio para $V$. guatemalensis.

Los mejores sitios, a partir de los valores de 0 a $20 \mathrm{~cm}$, están relacionados con $\mathrm{pH}$ de 5,2 , bajas concentraciones de $\mathrm{Ca}\left(3,89 \mathrm{cmol}(+) .1^{-1}\right)$ y concentraciones medias de $\mathrm{Mg}\left(1,31 \mathrm{cmol}(+) \cdot \mathrm{l}^{-1}\right)$, una $\Sigma$ Bases $\left(5,51 \mathrm{cmol}(+) .1^{-1}\right)$ y una CICE $(6,71$ $\left.\operatorname{cmol}(+) .1^{-1}\right)$ medias, una Ac.Extr. $\left(1,20 \mathrm{cmol}(+) \cdot \mathrm{l}^{-1}\right)$ media, con una SatAc media (18\%). Para los valores de 20 a $40 \mathrm{~cm}$, se observa que los mejores sitios están asociados con una baja DAp (1,14 g. $\left.\mathrm{cm}^{-3}\right)$, un suelo arcilloso Arc $(65,90 \%)$ y de $\mathrm{Fe}$ (166 mg. $\left.1^{-1}\right)$ y una buena cantidad de MO $(3,4 \%)$. Por lo tanto, se determina que $V$. guatemalensis tiene la capacidad de crecer y de ser más productiva bajo las condiciones mencionadas, lo que destaca los bajos requerimientos nutricionales que presenta la especie.

\section{RECOMENDACIONES}

Se deben validar los resultados del presente trabajo, para incluir una mayor variedad de condiciones ambientales regímenes de humedad del suelo, condiciones de fertilidad, condiciones fisiográficas, etc., al igual se recomienda ampliar el área geográfica de la investigación, 
para aumentar el tamaño de la muestra y mejorar la precisión de los resultados.

Con base en los resultados obtenidos a una profundidad de 0 a $20 \mathrm{~cm}$ es recomendable que $V$. guatemalensis se plante en suelos con valores de $\mathrm{pH} \leq 5,5$, contenidos de $\mathrm{Ca}$ y $\mathrm{Mg} \leq 5,65 \mathrm{cmol}(+) . \mathrm{l}^{-1}$ y $1,74 \mathrm{cmol}(+) \cdot \mathrm{l}^{-1}$, respectivamente una Ac. Extr. de $\geq 0,8 \mathrm{cmol}(+) .1^{-1}$ y una SatAc $\geq 11 \%$. Adicionalmente para la profundidad de 20 a $40 \mathrm{~cm}$ se recomiendan los valores que corresponden a una DAp $\leq 1,20$ g.cm ${ }^{-3}$, un contenido de Arc $\leq 72 \%$ y un contenido de $\mathrm{Fe} \geq 110 \mathrm{mg} \cdot \mathrm{l}^{-1}$.

\section{AGRADECIMIENTOS}

Muy especialmente a Marvin Castillo de la Escuela de Ingeniería Forestal del TEC, quien nos mostró los ensayos y facilitó la información dasométrica que se usó en este trabajo. Al compañero Henry Sánchez Toruño, quien colaboró en parte en las giras de campo y llevó a cabo los análisis de suelo en el Laboratorio de Análisis de Suelos y Foliares del Instituto de Investigación y Servicios Forestales, de la Universidad Nacional. Así mismo, a William Hernández y a Amelia Paniagua por su colaboración durante el trabajo de campo. A Mauricio Vega Araya por su colaboración en la confección del mapa de suelos. Y al maestro Alfredo Alvarado por sus comentarios a este documento.

\section{LITERATURA CITADA}

ALVARADO A., FALLAS J.L. 2004. Efecto de la saturación de acidez y el encalado sobre el crecimiento de la teca (Tectona grandis L.f.) en suelos ácidos de Costa Rica. Agronomía Costarricense 28(1):81-87.

ALVARADO A., RAIGOSA J. 2012. Nutrición y Fertilización Forestal en Regiones Tropicales. Asociación Costarricense de la Ciencia del Suelo. Editorama, S.A. San José, Costa Rica. 415 p.

ARIAS D., CALVO J., de B. RICHTER D., DOHRENBUSCH A. 2011. Productivity, aboveground biomass, nutrient uptake and carbon content in fast-growing tree plantations of native and introduced species in the southern region of Costa Rica. Biomass and Bioenergy 35(5): 1779-1788.

BADILLA Y. 2012. Concentración y absorción de elementos en plantaciones de Vochysia guatemalensis de las
Zonas Caribe y Norte de Costa Rica. Tesis de licenciatura, Instituto Tecnológico de Costa Rica, Cartago, Costa Rica. 76 p.

BAILEY R.L., CLUTTER J.L. 1974. Base-age invariant polymorphic site curves. Forest Science 20(2):155159.

BALZARINI M.G., GONZALEZ L., TABLADA M., CASANOVES F., DI RIENZO J.A., ROBLEDO C.W. 2008. INFOSTAT (Versión 2011e). Manual del Usuario, Editorial Brujas, Córdoba, Argentina.

BARROS N.F. de, WHITMORE J.L. 1980. Algunas consideraciones sobre relaciones entre sitio y suelo en los Neotrópicos. In: Producción de madera en los Neotrópicos por medio de plantaciones, actas de un simposio internacionál. Río Piedras, Puerto Rico. pp. 133-142.

BERTSCH F. 1986. Manual para interpretar la fertilidad de los suelos de Costa Rica. San José, Universidad de Costa Rica. 86 p.

BRICEÑO A.J., PACHECO R. 1984. Métodos Analíticos para el Estudio de Suelos y Plantas. Editorial de la Universidad de Costa Rica. San José, Costa Rica. $137 \mathrm{p}$.

BUOL S.W., HOLE F.D., McKRAKEN R.J. 1980. Soil genesis and classification. Ames, Iowa State University. 404 p.

BUTTERFIELD R. 1996. Early species selection for tropical reforestation: a consideration of stability. Forest Ecology and Management 81:161-168.

BUTTERFIELD R., ESPINOZA M. 1994. Screening trial of 14 tropical hardwoods with an emphasis on species native to Costa Rica: Fourth year results. New Forests 9:135-145.

CAB INTERNATIONAL 2000. Forestry Compendium Global Module. Wallingford, U. K. (disco compacto).

CATIE 1984. Taxonomía de suelos. Departamento de Producción Vegetál. Turrialba, Costa Rica. 311 p.

CLUTTER R., FORTSON J., PIENAAR L., BRISTER G., BAILEY R. 1983. Timber management: a quantitative approach. John Willey, New York. 333 p.

DAVIS L.S., JOHNSON K.N. 1987. Forest management. Third edition. McGraw-Hill, New York. 790 p.

DE HOOGH R.J., DIETRICH A.B. 1979. Avaliação de sítio para Araucaria angustifolia (Bert.) O. Ktze. em povoamentos artificiais. Brasil Florestal 10(37):19-71.

DELGADO A. 2002. Crecimiento de las plantaciones de especies nativas y su relación con la motivación de finqueros a reforestar en la Región Huetar Norte de Costa Rica. Informe de práctica de especialidad. Instituto Tecnológico de Costa Rica. Escuela de Ingeniería Forestal. Cartago, Costa Rica. 127 p.

DÍAZ ROMEO R., HUNTER A. 1982. Metodología de muestreo de Suelos, Análisis Químico de Suelos y Tejido Vegetal y de Investigación en Invernadero. CATIE. Turrialba, Costa Rica. 62 p. 
DI STEFANO J., FOURNIER L.A. 1994. Crecimiento inicial de Vochysia guatemalensis en Tabarcia de Mora, Costa Rica. Agronomía Costarricense 18(1):41-46.

DI STEFANO J., FOURNIER L.A. 1998. Biomasa aérea, concentración de nutrimentos y daños en árboles de Vochysia guatemalensis en un monocultivo de cuatro años, Tabarcia, Costa Rica. Agronomía Costarricense 22(2):235-241.

FASSBENDER H.W. 1975. Química de suelos con énfasis en suelos de América Latina. Instituto Interamericano de Ciencias Agrícolas. Turrialba, Costa Rica. 398 p.

FASSBENDER H.W., TSCHINKEL H. 1974. Relación entre el crecimiento de plantaciones de Cupressus lusitanica y las propiedades de los suelos derivados de cenizas volcánicas en Colombia. Turrialba 24(2):141-149.

FLORES E.M. 1993a. Vochysia guatemalensis, chancho blanco-white yemeri. Arboles y semillas del Neotrópico (Trees and seeds from the Neotropics) 2(2):1-27

GONZÁLEZ E., FISHER R.F. 1997. Variation in Foliar Elemental Composition in Mature Wild Trees and Among Families and Provenances of Vochysia guatemalensis in Costa Rica. Silvae Genetica 46(1):45-50.

HAGGAR J., RHEINGANS R., ARROYO P., ALVARADO B. 2003. Benefits and costs of intercropping reforestation in the Atlantic lowlands of Costa Rica. New Forests 25:41-48.

HARIDASAN M., MONTEIRO G. 1988. Aluminiumaccumulaing species in two forest communities in the Cerrado Region of Central Brazil. Forest Ecology and Management 24:15-26.

HARIDASAN M., PAVIANI T.I., SCHIAVINI Y. 1986. Localization of aluminium in the leaves of some aluminium-accumulating species. Plant and Soil 94:435-437.

HERRERA B. 1996. Evaluación del efecto de sitio en la productividad de las poblaciones de las dos especies dominantes en un bosque tropical de la tercera fase de la sucesión secundaria en Costa Rica. Tesis M.Sc. Turrialba, Costa Rica, Centro Agronómico Tropical de Investigación y Enseñanza. 152 p.

HERRERA B., CAMPOS J. 1999. Efecto de la variación del substrato y concentraciones foliares de nutrimentos en la distribución espacial y la productividad de Vochysia ferruginea en bosques tropicales secundarios de Costa Rica. XI Congreso Agronómico Nacional y de Recursos Naturales. Editorial Estatal a Distancia. San José, Costa Rica. 616 p.

HERRERA B., FINEGAN B. 1997. Substrate conditions, foliar nutrients and the distributions of two canopy tree species in a Costa Rican secondary rain forest. Plant and Soil 191:259-267.

JACKSON M.L. 1964. Soil Chemical Analysis. Prentice Hall Inc. Eglewood Cliffs, N.J. 498 p.
KAMPRATH E.J. 1967. Acidez del suelo y encalado. Boletín Técnico No. 4. International Soil Testing Program of North Carolina State. 20 p.

MOLLINEDO M., UGALDE L., ALVARADO A., VERJANS J.M., RUDY L.C. 2005. Relación sueloárbol y factores de sitio, en plantaciones jóvenes de teca (Tectona grandis), en la zona oeste de la cuenca del Canal de Panamá. Agronomía Costarricense 29(1):67-75.

MONTAGNINI F., SANCHO F. 1994. Net nitrogen mineralization in soils under six indigenous tree species, an abandoned pasture and a secondary forest in the Atlantic lowlands of Costa Rica. Plant and Soil 162:117-124.

MORA F. 1986. La clasificación de los rodales en relación con la calidad de sitio a partir de algunas características medibles. Ponencia, Primer Congreso Forestal Nacional, 10-14 de noviembre. San José, Costa Rica. 45 p.

MORA F. 1992. Criterios para clasificar los terrenos ocupados con plantaciones jóvenes de Teca (Tectona grandis Linn.). Ponencia Segundo Congreso Forestal Nacional, 25, 26 y 27 de noviembre. San José, Costa Rica. 125 p.

MORA F., MEZA V. 2003. Curvas de índice de sitio para Teca (Tectona grandis Linn.) en la Vertiente del Pacífico de Costa Rica. In: Plantaciones de Teca (Tectona grandis): Posibilidades y perspectivas para su desarrollo. Seminario, 26, 27 y 28 de Noviembre. Ed. INISEFOR. Heredia, Costa Rica. 16 p. 1 CD-ROM

MORA F., MEZA V. (sin publicar). Curvas de índice de sitio para Chancho (Vochysia guatemalensis) en la Región Huetar Norte de Costa Rica. Instituto de Investigación y Servicios Forestales. Universidad Nacional. Heredia, Costa Rica. 15 p.

MOREIRA I., ARNÁEZ E. 2007. Estudio radicular de Vochysia guatemalensis (cebo) en Sarapiquí, Heredia, Costa Rica. Tecnología en Marcha 19(1):311.

MOULAERT A., MUELLER J., VILLARREAL M., PIEDRA R., VILLALOBOS L. 2002. Establishment of two indigenous timber species in dairy pastures in Costa Rica. Agroforestry Systems 54:31-40.

OFI-CATIE (s.f.). Vochysiaceae, Vochysia guatemalensis Donn.Smith. Árboles de Centroamérica, pp. 947-950. Consultado en febrero 2012. Disponible en http:// www.herbaria.plants.ox.ac.uk/adc/ downloads/ capitulos_especies_y_anexos/vochysia_ guatemalensis.pdf

PÉREZ J., BORNEMIZSA E., SOLLINS P. 1993. Identificación de especies forestales acumuladoras de aluminio en una plantación forestal experimental ubicada en Sarapiquí, Costa Rica. Agronomía Costarricense 17(2):99-103. 
PHILIP M.S. 1983. Measuring trees and forests. A textbook written for students in Africa. Dept. of For. Univ. Aberdeen, Aberdeen, UK. 338 p.

PIOTTO D., CRAVEN D., MONTAGNINI F., ALICE F. 2010. Silvicultural and economic aspects of pure and mixed native tree species plantations on degraded pasturelands in humid Costa Rica. New Forests 39:369-385.

PRODAN M., PETERS R., COX F., REAL P. 1997. Mensura Forestal. IICA-GTZ. San José, Costa Rica. 561 p.

SAMPAT A. 1979. Física de Suelos, Principios y Aplicaciones. Editorial Limusa. México. 351 p.

SOLÍS M., MOYA R. 2004. Vochysia guatemalensis en Costa Rica. Fondo Nacional de Financiamiento Forestal.
Proyecto Reforesta. Banco Mundiál. Consultado enero 2012. Disponible en http://www.fonafifo.com

TUSELL F. 2012. Análisis Multivariante. Notas del curso Estadística: Análisis Multivariante. Capítulo 7: Biplots. Licenciatura en Sociología. Facultad de Ciencias Políticas y Sociología. Universidad de Granada, España. 164 p. Consultado febrero 2013. Disponible en http://www.et.bs.ehu.es/ etptupaf/ nuevo/ficheros/estad4/multi.pdf Also In: http://www. ugr.es/ ramongs/guiamultivariante.pdf

WOODRUFF J.R., KAMPRATH F.J. 1965. Phosphorus adsorption maximum as measured by the Langmuir isotherm and its relationship to phosphorus availability. Soil Sci. Soc. Amer. Proc. 29:148-150. 
